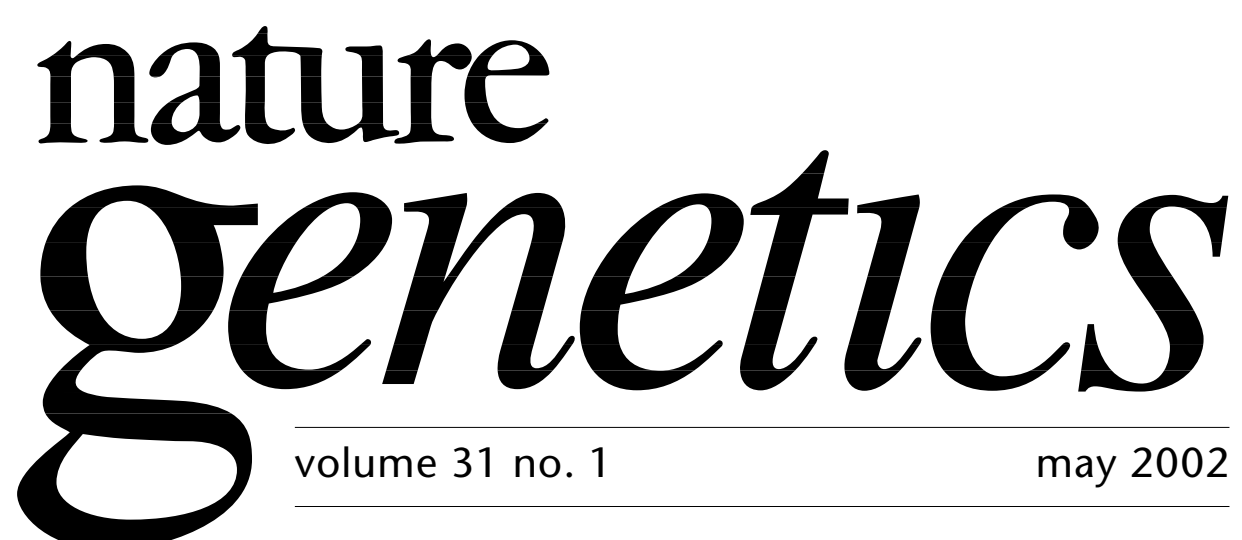

\title{
Gene expression and cancer: getting it together
}

A recent article in The New York Times, "Telling Threatening Tumors from Harmless Ones," alerted its readers that "it may be possible to spare women needless treatments by taking a genetic fingerprint of their tumors." The article was referring to a study published earlier this year in Nature that linked genome-wide expression profiles of breast cancer samples to clinical outcome. The study implied that, at least for some cancers, molecular classification schemes might be superior to current histopathological and clinical criteria in determining course of treatment and could dramatically improve patient care-a prospect that has obviously grabbed media and public attention.

It's too early to say whether such expectations will be met and when. Nonetheless, it seems likely that high-throughput gene expression analyses will accelerate the rate of discovery in cancer biology and the identification of possible therapeutic targets. But as gene-expression data sets have begun to materialize, so have the challenges of interpreting them. A major pitfall is that there are no standard methodologies or vocabulary for DNA expression studies, making it difficult to compare results from one to another-and indeed results often appear inconsistent. Another problem is the typically small number of samples analyzed in a given study. For example, the authors of the breast cancer study picked up by The Times were able to correctly classify 17 of 19 cancer samples according to their metastatic potential. It remains to be seen whether the same strategy applied to a large patient cohort will reveal equally impressive predictive potential. In this respect, the availability of a large collection of carefully obtained and annotated tumor material analyzed using standardized methods would be a powerful resource.

Enter the International Genomics Consortium (IGC). The IGC was formed about a year and a half ago, with the goal of obtaining and carrying out gene expression analyses on a highly annotated set of tumor samples and making the information available in a database - the largest of its kind - to all researchers. The first round of expression profiling of brain cancer samples was recently completed in temporary laboratory quarters provided by Scottsdale Healthcare in Arizona. If the pilot project convinces prospective funders to provide the requested $\$ 42$ million, and medical centers and hospitals to supply the required tumor samplesand providing that remaining procedural issues and ethical concerns can be worked through — the project should launch in the fall of 2003. 
The idea of a cancer gene expression database is not new, and several large cancer centers and private companies are undertaking similar projects-but the proposed scale and level of access provided by the IGC stand out. One of its goals is to carry out gene expression profiling of a broad sampling of 10,000 cancer samples, as well as 1,000 normal tissues, over a three-year period. The data, along with clinical information obtained at the point of acquisition of the tumor and from longitudinal tracking of patients' outcome (done in way to preserve their anonymity), will populate the publicly available IGC database with regularly scheduled releases, and there will be no restrictions on its use. Excess samples will be embedded into tissue microarray blocks for storage and further study.

Another notable feature of the IGC database will be the implementation of standardized procedures at each step of the process. The variation in experimental conditions and analytical tools used make it difficult to integrate expression data from different experiments in a central database. The IGC will therefore carry out hybridization experiments at a central facility-possibly at Scottsdale Healthcare, where the pilot test was run-using identical conditions and reagents. The database will implement representation standards and defined data structures, consistent with those proposed by the MGED group, to allow unambiguous integration of information.

Although three million biopsy tumor samples (or thereabouts) are generated each year, obtaining a large set of high quality, annotated samples with appropriate clinical follow-up and patient consent is a challenge for most investigators. Aside from practical limitations, such as obtaining samples of sufficient quality in terms of RNA integrity, fixation or accompanying patient information, the process has both ethical and cost implications. Whereas researchers at large cancer centers have access to tumor samples and patients, it would be difficult to collect samples on the scale envisioned by IGC and at the same time, dedicate resources and money required to analyze them-the challenge is insurmountable for smaller centers that may not even have bioinformatics facilities. Industry, on the other hand, has great platforms for analyzing data but no access to patients.

The IGC represents a marriage between academic centers and industry; its spawn, a public resource that will benefit both. It has established partnerships with several academic medical centers and hospitals (so far, all in the United States, but the plan is to eventually draw in centers from around the world) that will provide surgical specimens according to a standardized procedure for collection and annotation and in accordance with ethical requirements. Importantly, samples will be stripped of any information that would reveal a patient's identity, but a mechanism will be put in place to allow the retrieval of information about how a patient responds to treatment and possibly_although this is still far off_-relaying medically useful information back to patients. Reagents for the experiments and tools for analyzing them will be provided by companies like Affymetrix, Agilent Technologies, and Motorola (which have sponsored the pilot project).

According to IGC organizers, fulfilling the ethical and legal requirements of obtaining human tissues and patients' consent has been tough. Whether a sufficient number patients will consent to providing cancer samples is still an open question-and one on which the ability of IGC to get its project off the ground critically depends. If successful, the groundwork laid out by IGC will serve as a model for other kinds of tissue or array expression banks.

Whether the day will come when, as The New York Times article predicts, "instead of saying a person has lung cancer, it might be said the person has cancer with particular generic characteristics that just happens to be in the lung," remains to be seen. The IGC is taking a bold step toward understanding the true potential and promise of genomics research.

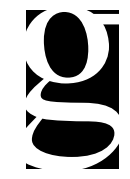

